\title{
SOME NEW INEQUALITIES FOR DIFFERENTIABLE $h$-CONVEX FUNCTIONS AND APPLICATIONS
}

\author{
MUSA ÇAKMAK, MEVLÜT TUNÇ, AND AYŞEGÜL ACEM \\ Received 06 November, 2017
}

\begin{abstract}
In this paper, the authors established a new identity for differentiable functions, afterwards they obtained some new inequalities for functions whose first derivatives in absolute value at certain powers are $h$-convex by using the identity. Also they give some applications for special means for arbitrary positive numbers.
\end{abstract}

2010 Mathematics Subject Classification: 26A15; 26D07; 26D08

Keywords: $h$-convex function, $s$-convex function, $\operatorname{tgs}$-convex functions, Hadamard inequality, Hölder inequality

\section{INTRODUCTION}

\subsection{Definitions}

A function $f: I \rightarrow R, I \subseteq R$ is an interval, is said to be a convex function on $I$ if

$$
f(t x+(1-t) y) \leq t f(x)+(1-t) f(y)
$$

holds for all $x, y \in I$ and $t \in[0,1]$. If the reversed inequality in (1.1) holds, then $f$ is concave.

We say that $f: I \rightarrow \mathbb{R}$ is Godunova-Levin function or that $f$ belongs to the class $Q(I)$ if $f$ is nonnegative and for all $x, y \in I$ and $t \in(0,1)$ we have

$$
f(t x+(1-t) y) \leq \frac{f(x)}{t}+\frac{f(y)}{1-t}
$$

[13, Godunova and Levin, 1985].

Let $s \in(0,1]$. A function $f:(0, \infty] \rightarrow[0, \infty]$ is said to be $s$-convex in the second sense if

$$
f(t x+(1-t) y) \leq t^{s} f(x)+(1-t)^{s} f(y),
$$

for all $x, y \in(0, \infty]$ and $t \in[0,1]$. This class of $s$-convex functions is usually denoted by $K_{s}^{2}$ [14, Hudzik and Maligranda, 1994].

In 1978, Breckner introduced $s$-convex functions as a generalization of convex functions in [6]. Also, in that work Breckner proved the important fact that the set valued map is $s$-convex only if the associated support function is $s$-convex function in 
[7]. A number of properties and connections with $s$-convex in the first sense and its generalizations are discussed in the papers $[9,10,14]$. Of course, $s$-convexity means just convexity when $s=1$.

We say that $f: I \rightarrow \mathbb{R}$ is a $P$-function or that $f$ belongs to the class $P(I)$ if $f$ is nonnegative and for all $x, y \in I$ and $t \in[0,1]$, we have

$$
f(t x+(1-t) y) \leq f(x)+f(y)
$$

[12, Dragomir, Pečarić and Persson, 1995].

Let $h: J \rightarrow \mathbb{R}$ be a nonnegative function, $h \not \equiv 0$. We say that $f: I \rightarrow \mathbb{R}$ is an $h$ convex function, or that $f$ belongs to the class $S X(h, I)$, if $f$ is nonnegative and for all $x, y \in I$ and $t \in(0,1)$ we have

$$
f(t x+(1-t) y) \leq h(t) f(x)+h(1-t) f(y) .
$$

If inequality (1.5) is reversed, then $f$ is said to be $h$-concave, i.e. $f \in S V(h, I)$. Obviously, if $h(t)=t$, then all nonnegative convex functions belong to $S X(h, I)$ and all nonnegative concave functions belong to $S V(h, I)$; if $h(t)=\frac{1}{t}$, then $S X(h, I)=$ $Q(I)$; if $h(t)=1$, then $S X(h, I) \supseteq P(I)$; and if $h(t)=t^{s}$, where $s \in(0,1)$, then $S X(h, I) \supseteq K_{s}^{2}$ [22, Varošanec, 2007].

A function $f: I \subseteq \mathbb{R} \rightarrow \mathbb{R}$ is said to belong to the class of $M T(I)$ if it is nonnegative and for all $x, y \in I$ and $t \in(0,1)$ satisfies the inequality;

$$
f(t x+(1-t) y) \leq \frac{\sqrt{t}}{2 \sqrt{1-t}} f(x)+\frac{\sqrt{1-t}}{2 \sqrt{t}} f(y)
$$

[21, Tunç and Yıldırım, 2012]. Definition of $M T$-convex function may be regarded as a special case of $h$-convex function. And in (1.6), if we take $t=1 / 2$, inequality (1.6) reduces to Jensen convex.

Let $f: I \subset \mathbb{R} \rightarrow \mathbb{R}$ be a nonnegative function. We say that $f: I \rightarrow \mathbb{R}$ is $\operatorname{tg} s-$ convex function on $I$ if the inequality

$$
f(t x+(1-t) y) \leq t(1-t)[f(x)+f(y)]
$$

holds for all $x, y \in I$ and $t \in(0,1)$. We say that $f$ is $\operatorname{tg} s$-concave if $(-f)$ is $\operatorname{tg} s$-convex [20]. In (1.5), if we take $h(t)=t(1-t)$, inequality (1.5) reduces to inequality (1.7).

\subsection{Theorems}

If $f$ is integrable on $[a, b]$, then the average value of $f$ on $[a, b]$ is

$$
\frac{1}{b-a} \int_{a}^{b} f(x) d x \text {. }
$$

Let $f: I \subseteq \mathbb{R} \rightarrow \mathbb{R}$ be a convex function and $a, b \in I$ with $a<b$. Then the following double inequality:

$$
f\left(\frac{a+b}{2}\right) \leq \frac{1}{b-a} \int_{a}^{b} f(x) d x \leq \frac{f(a)+f(b)}{2}
$$


is known as Hermite-Hadamard inequality for convex mappings. For particular choice of the function $f$ in (1.8) yields some classical inequalities of means. Both inequalities in (1.8) hold in reversed direction if $f$ is concave. The refinement of the second inequality in (1.8) is due to Bullen as follows:

$$
\frac{1}{b-a} \int_{a}^{b} f(x) d x \leq \frac{1}{2}\left[f\left(\frac{a+b}{2}\right)+\frac{f(a)+f(b)}{2}\right] \leq \frac{f(a)+f(b)}{2}
$$

where $f$ is as above. This (1.9) integral inequality is well known in the literature as Bullen Inequality [18, Pečarić, Proschan and Tong, 1991]. For some recent results in connection with Hermite-Hadamard inequality and its applications we refer to $[1-5,12,15,16,21,22]$ where further references are given.

The following inequality is well known in the literature as Simpson's inequality [11, Dragomir, Agarwal, and Cerone, 2000];

$$
\int_{a}^{b} f(x) d x-\frac{b-a}{3}\left[\frac{f(a)+f(b)}{2}+2 f\left(\frac{a+b}{2}\right)\right] \leq \frac{1}{1280}\left\|f^{(4)}\right\|_{\infty}(b-a)^{5},
$$

where the mapping $f:[a, b] \rightarrow \mathbb{R}$ is assumed to be four times continuously differentiable on the interval and $f^{(4)}$ to be bounded on $(a, b)$, that is,

$$
\left\|f^{(4)}\right\|_{\infty}=\sup _{t \in(a, b)}\left|f^{(4)}(t)\right|<\infty .
$$

In [19], M. Z. Sarıkaya, A. Sağlam and H. Yıldırım established the following Hadamard type inequality for $h$-convex functions:

Let $f \in S X(h, I), a, b \in I$ and $f \in L_{1}([a, b])$, then

$$
\frac{1}{2 h\left(\frac{1}{2}\right)} f\left(\frac{a+b}{2}\right) \leq \frac{1}{b-a} \int_{a}^{b} f(x) d x \leq[f(a)+f(b)] \int_{0}^{1} h(t) d t .
$$

For recent results and generalizations concerning $h$-convex functions see $[5,8,17$, 19,22] and references therein.

In this paper, firstly we will derive a new general inequality for functions whose first derivatives in absolute value are $h$-convex, which not only provides a generalization of the previous theorems but also gives some other interesting special results. Then we give some corollaries and remarks for different type convex functions. Finally, applications to some special means of real numbers are considered.

\section{MAin Results}

Lemma 1. Let $f: I \subset R \rightarrow R$ be a differentiable function on $I^{\circ}$ such that $f^{\prime} \in$ $L^{1}[a, b]$, where $a, b \in I$ with $a<b$. Then, for any $\varepsilon \in[0,1]$, the following equality holds:

$$
(1-2 \varepsilon) f\left(\frac{a+b}{2}\right)+\varepsilon[f(a)+f(b)]-\frac{1}{b-a} \int_{a}^{b} f(x) d x
$$


$=\frac{b-a}{4}\left\{\int_{0}^{1}(t-2 \varepsilon) f^{\prime}\left(t \frac{a+b}{2}+(1-t) a\right) d t+\int_{0}^{1}(2 \varepsilon-t) f^{\prime}\left(t \frac{a+b}{2}+(1-t) b\right) d t\right\}$

Proof. Integrating by parts, we have the following identity:

$$
\begin{aligned}
I_{1} & =\int_{0}^{1}(t-2 \varepsilon) f^{\prime}\left(t \frac{a+b}{2}+(1-t) a\right) d t \\
& =\left.(t-2 \varepsilon) \frac{2}{b-a} f\left(t \frac{a+b}{2}+(1-t) a\right)\right|_{0} ^{1}-\frac{2}{b-a} \int_{0}^{1} f\left(t \frac{a+b}{2}+(1-t) a\right) d t \\
& =\frac{2(1-2 \varepsilon)}{b-a} f\left(\frac{a+b}{2}\right)+\frac{4 \varepsilon}{b-a} f(a)-\frac{2}{b-a} \int_{0}^{1} f\left(t \frac{a+b}{2}+(1-t) a\right) d t .
\end{aligned}
$$

Using the change of variable $x=t \frac{a+b}{2}+(1-t) a$ for $t \in[0,1]$ and multiplying both sides of (2.2) by $\frac{b-a}{4}$, we obtain

$$
\begin{aligned}
\frac{b-a}{4} \int_{0}^{1}(t-2 \varepsilon) f^{\prime}(t & \left.\frac{a+b}{2}+(1-t) a\right) d t \\
& =\frac{1-2 \varepsilon}{2} f\left(\frac{a+b}{2}\right)+\varepsilon f(a)-\frac{1}{b-a} \int_{a}^{\frac{a+b}{2}} f(x) d x
\end{aligned}
$$

Similarly, we observe that

$$
\begin{aligned}
& I_{2}=\int_{0}^{1}(2 \varepsilon-t) f^{\prime}\left(t \frac{a+b}{2}+(1-t) b\right) d t \\
& =\frac{2(2 \varepsilon-1)}{a-b} f\left(\frac{a+b}{2}\right)-\frac{4 \varepsilon}{a-b} f(b)+\frac{2}{a-b} \int_{0}^{1} f\left(t \frac{a+b}{2}+(1-t) b\right) d t .
\end{aligned}
$$

Using the change of variable $x=t \frac{a+b}{2}+(1-t) b$ for $t \in[0,1]$ and multiplying both sides of (2.4) by $\frac{b-a}{4}$, we obtain

$$
\begin{aligned}
\frac{b-a}{4} \int_{0}^{1}(2 \varepsilon-t) f^{\prime}\left(t \frac{a+b}{2}+(1-t) b\right) d t & \\
& =\frac{1-2 \varepsilon}{2} f\left(\frac{a+b}{2}\right)+\varepsilon f(b)-\frac{1}{b-a} \int_{\frac{a+b}{2}}^{b} f(x) d x
\end{aligned}
$$

Thus, adding (2.3) and (2.5), we get the required identity (2.1).

Theorem 1. Let $I \subset[0, \infty), f: I \rightarrow R$ be a differentiable function on $I^{\circ}$ such that $f^{\prime} \in L^{1}[a, b]$, where $a, b \in I$ with $a<b$. If $\left|f^{\prime}\right|^{q}$ is $h$-convex on $[a, b]$ for some fixed 
$t \in(0,1)$ and $q \geq 1$, then the following inequalities hold

$$
\begin{aligned}
& \left|(1-2 \varepsilon) f\left(\frac{a+b}{2}\right)+\varepsilon[f(a)+f(b)]-\frac{1}{b-a} \int_{a}^{b} f(x) d x\right| \\
& \leq \frac{b-a}{4}\left(4 \varepsilon^{2}-2 \varepsilon+\frac{1}{2}\right)^{1-\frac{1}{q}} \\
& \quad \times\left[\left\{\left|f^{\prime}\left(\frac{a+b}{2}\right)\right|^{q} \int_{0}^{1}|2 \varepsilon-t| h(t) d t+\left|f^{\prime}(a)\right|^{q} \int_{0}^{1}|2 \varepsilon-t| h(1-t) d t\right\}^{\frac{1}{q}}\right. \\
& \left.+\left\{\left|f^{\prime}\left(\frac{a+b}{2}\right)\right|^{q} \int_{0}^{1}|2 \varepsilon-t| h(t) d t+\left|f^{\prime}(b)\right|^{q} \int_{0}^{1}|2 \varepsilon-t| h(1-t) d t\right\}^{\frac{1}{q}}\right]
\end{aligned}
$$

for $0 \leq \varepsilon \leq \frac{1}{2}$, and

$$
\begin{aligned}
\mid & (1-2 \varepsilon) f\left(\frac{a+b}{2}\right)+\varepsilon[f(a)+f(b)]-\frac{1}{b-a} \int_{a}^{b} f(x) d x \mid \\
\leq & \frac{b-a}{4}\left(2 \varepsilon-\frac{1}{2}\right){ }^{1-\frac{1}{q}} \\
& \times\left[\left\{\left|f^{\prime}\left(\frac{a+b}{2}\right)\right|^{q} \int_{0}^{1}|2 \varepsilon-t| h(t) d t+\left|f^{\prime}(a)\right|^{q} \int_{0}^{1}|2 \varepsilon-t| h(1-t) d t\right\}^{\frac{1}{q}}\right. \\
& \left.+\left\{\left|f^{\prime}\left(\frac{a+b}{2}\right)\right|^{q} \int_{0}^{1}|2 \varepsilon-t| h(t) d t+\left|f^{\prime}(b)\right|^{q} \int_{0}^{1}|2 \varepsilon-t| h(1-t) d t\right\}^{\frac{1}{q}}\right]
\end{aligned}
$$

for $\frac{1}{2} \leq \varepsilon \leq 1$.

Proof. In case $0 \leq \varepsilon \leq \frac{1}{2}$, by Lemma 1 and using the Hölder inequality, we have

$$
\begin{aligned}
& \left|(1-2 \varepsilon) f\left(\frac{a+b}{2}\right)+\varepsilon[f(a)+f(b)]-\frac{1}{b-a} \int_{a}^{b} f(x) d x\right| \\
& \leq \frac{b-a}{4}\left\{\left(\int_{0}^{1}|t-2 \varepsilon| d t\right)^{1-\frac{1}{q}}\left(\int_{0}^{1}|t-2 \varepsilon|\left|f^{\prime}\left(t \frac{a+b}{2}+(1-t) a\right)\right|^{q} d t\right)^{\frac{1}{q}}\right. \\
& \left.\quad+\left(\int_{0}^{1}|2 \varepsilon-t| d t\right)^{1-\frac{1}{q}}\left(\int_{0}^{1}|2 \varepsilon-t|\left|f^{\prime}\left(t \frac{a+b}{2}+(1-t) b\right)\right|^{q} d t\right)^{\frac{1}{q}}\right\} \\
& \leq \frac{b-a}{4}\left(4 \varepsilon^{2}-2 \varepsilon+\frac{1}{2}\right)^{1-\frac{1}{q}}
\end{aligned}
$$




$$
\begin{aligned}
& \times\left[\left\{\left|f^{\prime}\left(\frac{a+b}{2}\right)\right|^{q} \int_{0}^{1}|2 \varepsilon-t| h(t) d t+\left|f^{\prime}(a)\right|^{q} \int_{0}^{1}|2 \varepsilon-t| h(1-t) d t\right\}^{\frac{1}{q}}\right. \\
& \left.+\left\{\left|f^{\prime}\left(\frac{a+b}{2}\right)\right|^{q} \int_{0}^{1}|2 \varepsilon-t| h(t) d t+\left|f^{\prime}(b)\right|^{q} \int_{0}^{1}|2 \varepsilon-t| h(1-t) d t\right\}^{\frac{1}{q}}\right],
\end{aligned}
$$

where

$$
\int_{0}^{1}|t-2 \varepsilon| d t=4 \varepsilon^{2}-2 \varepsilon+\frac{1}{2} .
$$

In case $\frac{1}{2} \leq \varepsilon \leq 1$, by Lemma 1 and using the Hölder inequality, we have

$$
\begin{aligned}
\mid & (1-2 \varepsilon) f\left(\frac{a+b}{2}\right)+\varepsilon[f(a)+f(b)]-\frac{1}{b-a} \int_{a}^{b} f(x) d x \mid \\
\leq & \frac{b-a}{4}\left[\left(\int_{0}^{1}|t-2 \varepsilon| d t\right)^{1-\frac{1}{q}}\left\{\int_{0}^{1}|t-2 \varepsilon|\left|f^{\prime}\left(t \frac{a+b}{2}+(1-t) a\right)\right|^{q} d t\right\}^{\frac{1}{q}}\right. \\
& \left.+\left(\int_{0}^{1}|2 \varepsilon-t| d t\right)^{1-\frac{1}{q}}\left(\int_{0}^{1}|2 \varepsilon-t|\left|f^{\prime}\left(t \frac{a+b}{2}+(1-t) b\right)\right|^{q} d t\right)^{\frac{1}{q}}\right] \\
\leq & \left.\frac{b-a}{4}\left(2 \varepsilon-\frac{1}{2}\right)\right]^{1-\frac{1}{q}} \\
& \times\left[\left\{\left|f^{\prime}\left(\frac{a+b}{2}\right)\right|^{q} \int_{0}^{1}|2 \varepsilon-t| h(t) d t+\left|f^{\prime}(a)\right|^{q} \int_{0}^{1}|2 \varepsilon-t| h(1-t) d t\right\}^{\frac{1}{q}}\right. \\
& \left.+\left\{\left|f^{\prime}\left(\frac{a+b}{2}\right)\right|^{q} \int_{0}^{1}|2 \varepsilon-t| h(t) d t+\left|f^{\prime}(b)\right|^{q} \int_{0}^{1}|2 \varepsilon-t| h(1-t) d t\right\}^{\frac{1}{q}}\right],
\end{aligned}
$$

where

Thus, the proof is completed.

$$
\int_{0}^{1}|t-2 \varepsilon| d t=2 \varepsilon-\frac{1}{2}
$$

Corollary 1. Let $I \subset[0, \infty), f: I \rightarrow R$ be a differentiable function on $I^{\circ}$ such that $f^{\prime} \in L^{1}[a, b]$, where $a, b \in I$ with $a<b$. If $\left|f^{\prime}\right|^{q}$ is $h$-convex on $[a, b]$ for some fixed $t \in(0,1)$ and $q=1$, then the following inequalities hold

$$
\begin{aligned}
& \left|(1-2 \varepsilon) f\left(\frac{a+b}{2}\right)+\varepsilon[f(a)+f(b)]-\frac{1}{b-a} \int_{a}^{b} f(x) d x\right| \\
& \leq \frac{b-a}{4}\left[2\left|f^{\prime}\left(\frac{a+b}{2}\right)\right| \int_{0}^{1}|2 \varepsilon-t| h(t) d t+\left(\left|f^{\prime}(b)\right|+\left|f^{\prime}(a)\right|\right) \int_{0}^{1}|2 \varepsilon-t| h(1-t) d t\right]
\end{aligned}
$$

for $0 \leq \varepsilon \leq 1$. 
Proof. Inequalities (2.8) is immediate by setting $q=1$ in (2.6) and (2.7) of Theorem 1.

Remark 1 . If we take $\varepsilon=0$ in (2.8) then we get a midpoint type inequality

$$
\begin{aligned}
& \left|\frac{1}{b-a} \int_{a}^{b} f(x) d x-f\left(\frac{a+b}{2}\right)\right| \\
& \quad \leq \frac{b-a}{4}\left[2\left|f^{\prime}\left(\frac{a+b}{2}\right)\right| \int_{0}^{1} t h(t) d t+\left(\left|f^{\prime}(a)\right|+\left|f^{\prime}(b)\right|\right) \int_{0}^{1} t h(1-t) d t\right] .
\end{aligned}
$$

If we take $\varepsilon=\frac{1}{2}$ in (2.8), then we get a trapezoid type inequality

$$
\begin{aligned}
\left|\frac{1}{b-a} \int_{a}^{b} f(x) d x-\frac{f(a)+f(b)}{2}\right| \leq & \frac{b-a}{4}\left[2\left|f^{\prime}\left(\frac{a+b}{2}\right)\right|\left(\int_{0}^{1}|t-1| h(t) d t\right)\right. \\
& \left.+\left(\left|f^{\prime}(a)\right|+\left|f^{\prime}(b)\right|\right)\left(\int_{0}^{1}|t-1| h(1-t) d t\right)\right] .
\end{aligned}
$$

If we take $\varepsilon=\frac{1}{4}$ in (2.8), then we get a Bullen type inequality

$$
\begin{aligned}
& \mid \frac{1}{b-a} \int_{a}^{b} f(x) d x-\frac{1}{4}[f(a)\left.+2 f\left(\frac{a+b}{2}\right)+f(b)\right] \mid \\
& \leq \frac{b-a}{4}\left\{2\left|f^{\prime}\left(\frac{a+b}{2}\right)\right|\left(\int_{0}^{1}\left|t-\frac{1}{2}\right| h(t) d t\right)\right. \\
&\left.+\left(\left|f^{\prime}(a)\right|+\left|f^{\prime}(b)\right|\right)\left(\int_{0}^{1}\left|t-\frac{1}{2}\right| h(1-t) d t\right)\right\} .
\end{aligned}
$$

If we take $\varepsilon=\frac{1}{6}$ in (2.8), then we get a Simpson type inequality

$$
\begin{aligned}
& \mid \frac{1}{b-a} \int_{a}^{b} f(x) d x-\frac{1}{6}[f(a)\left.+4\left(\frac{a+b}{2}\right)+f(b)\right] \mid \\
& \leq \frac{b-a}{4}\left\{2\left|f^{\prime}\left(\frac{a+b}{2}\right)\right|\left(\int_{0}^{1}\left|t-\frac{1}{3}\right| h(t) d t\right)\right. \\
&\left.+\left(\left|f^{\prime}(a)\right|+\left|f^{\prime}(b)\right|\right)\left(\int_{0}^{1}\left|t-\frac{1}{3}\right| h(1-t) d t\right)\right\} .
\end{aligned}
$$

Corollary 2. Under the assumption of Theorem 1, if $\left|f^{\prime}\right|^{q}$ is s-convex in the second sense on $[a, b]$ for some fixed $s \in(0,1]$ and $q \geq 1$, then the following inequalities hold: 


$$
\begin{aligned}
& \left|(1-2 \varepsilon) f\left(\frac{a+b}{2}\right)+\varepsilon[f(a)+f(b)]-\frac{1}{b-a} \int_{a}^{b} f(x) d x\right| \\
& \leq \frac{b-a}{4}\left(4 \varepsilon^{2}-2 \varepsilon+\frac{1}{2}\right)^{1-\frac{1}{q}}\left[\left(\left|f^{\prime}\left(\frac{a+b}{2}\right)\right|^{q} P(s, \varepsilon)+\left|f^{\prime}(a)\right|^{q} Q(s, \varepsilon)\right)^{\frac{1}{q}}\right. \\
& \left.+\left(\left|f^{\prime}\left(\frac{a+b}{2}\right)\right|^{q} P(s, \varepsilon)+\left|f^{\prime}(b)\right|^{q} Q(s, \varepsilon)\right)^{\frac{1}{q}}\right]
\end{aligned}
$$

for $0 \leq \varepsilon \leq \frac{1}{2}$, where $P(s, \varepsilon)=\frac{s-4 \varepsilon-2 s \varepsilon+2(2 \varepsilon)^{s+2}+1}{(s+1)(s+2)}, Q(s, \varepsilon)=\frac{2(1-2 \varepsilon)^{s+2}+4 \varepsilon+2 s \varepsilon-1}{(s+1)(s+2)}$, and

$$
\begin{aligned}
\mid(1-2 \varepsilon) f\left(\frac{a+b}{2}\right)+ & \varepsilon[f(a)+f(b)]-\frac{1}{b-a} \int_{a}^{b} f(x) d x \mid \\
\leq \frac{b-a}{4}\left(2 \varepsilon-\frac{1}{2}\right)^{1-\frac{1}{q}} & {\left[\left(\left|f^{\prime}\left(\frac{a+b}{2}\right)\right|^{q} U(s, \varepsilon)+\left|f^{\prime}(a)\right|^{q} V(s, \varepsilon)\right)^{\frac{1}{q}}\right.} \\
& \left.+\left(\left|f^{\prime}\left(\frac{a+b}{2}\right)\right|^{q} U(s, \varepsilon)+\left|f^{\prime}(b)\right|^{q} V(s, \varepsilon)\right)^{\frac{1}{q}}\right]
\end{aligned}
$$

for $\frac{1}{2} \leq \varepsilon \leq 1$ where $U(s, \varepsilon)=\frac{2 \varepsilon(s+2)-(s+1)}{(s+1)(s+2)}, V(s, \varepsilon)=\frac{4 \varepsilon+2 s \varepsilon-1}{(s+1)(s+2)}$.

Proof. In case $0 \leq \varepsilon \leq \frac{1}{2}$, by Lemma 1 and using the Hölder inequality, we have

$$
\begin{aligned}
\mid & (1-2 \varepsilon) f\left(\frac{a+b}{2}\right)+\varepsilon[f(a)+f(b)]-\frac{1}{b-a} \int_{a}^{b} f(x) d x \mid \\
\leq & \frac{b-a}{4}\left[\left(\int_{0}^{1}|t-2 \varepsilon| d t\right)^{1-\frac{1}{q}}\left(\int_{0}^{1}|t-2 \varepsilon|\left|f^{\prime}\left(t \frac{a+b}{2}+(1-t) a\right)\right|^{q} d t\right)^{\frac{1}{q}}\right. \\
& \left.+\left(\int_{0}^{1}|2 \varepsilon-t| d t\right)^{1-\frac{1}{q}}\left(\int_{0}^{1}|2 \varepsilon-t|\left|f^{\prime}\left(t \frac{a+b}{2}+(1-t) b\right)\right|^{q} d t\right)^{\frac{1}{q}}\right] \\
\leq & \frac{b-a}{4}\left(4 \varepsilon^{2}-2 \varepsilon+\frac{1}{2}\right)^{1-\frac{1}{q}}\left[\left(\int_{0}^{1}|2 \varepsilon-t|\left(t^{s}\left|f^{\prime}\left(\frac{a+b}{2}\right)\right|^{q}+(1-t)^{s}\left|f^{\prime}(a)\right|^{q}\right) d t\right)^{\frac{1}{q}}\right. \\
& \left.+\left(\int_{0}^{1}|2 \varepsilon-t|\left(t^{s}\left|f^{\prime}\left(\frac{a+b}{2}\right)\right|^{q}+(1-t)^{s}\left|f^{\prime}(b)\right|^{q}\right) d t\right)^{\frac{1}{q}}\right] \\
= & \frac{b-a}{4}\left(4 \varepsilon^{2}-2 \varepsilon+\frac{1}{2}\right)^{1-\frac{1}{q}}\left[\left\{\int_{0}^{2 \varepsilon}(2 \varepsilon-t)\left(t^{s}\left|f^{\prime}\left(\frac{a+b}{2}\right)\right|^{q}+(1-t)^{s}\left|f^{\prime}(a)\right|^{q}\right) d t\right.\right.
\end{aligned}
$$




$$
\begin{aligned}
& \left.+\int_{2 \varepsilon}^{1}(t-2 \varepsilon)\left(t^{s}\left|f^{\prime}\left(\frac{a+b}{2}\right)\right|^{q}+(1-t)^{s}\left|f^{\prime}(a)\right|^{q}\right) d t\right\}^{\frac{1}{q}} \\
& +\left\{\int_{0}^{2 \varepsilon}(2 \varepsilon-t)\left(t^{s}\left|f^{\prime}\left(\frac{a+b}{2}\right)\right|^{q}+(1-t)^{s}\left|f^{\prime}(b)\right|^{q}\right) d t\right. \\
& \left.\left.+\int_{2 \varepsilon}^{1}(t-2 \varepsilon)\left(t^{s}\left|f^{\prime}\left(\frac{a+b}{2}\right)\right|^{q}+(1-t)^{s}\left|f^{\prime}(b)\right|^{q}\right) d t\right\}^{\frac{1}{q}}\right] \\
& =\frac{b-a}{4}\left(4 \varepsilon^{2}-2 \varepsilon+\frac{1}{2}\right)^{1-\frac{1}{q}} \\
& \times\left[\left(\left|f^{\prime}\left(\frac{a+b}{2}\right)\right|^{q} \frac{s-4 \varepsilon-2 s \varepsilon+2(2 \varepsilon)^{s+2}+1}{(s+1)(s+2)}+\left|f^{\prime}(a)\right|^{q} \frac{2(1-2 \varepsilon)^{s+2}+4 \varepsilon+2 s \varepsilon-1}{(s+1)(s+2)}\right)^{\frac{1}{q}}\right. \\
& \left.+\left(\left|f^{\prime}\left(\frac{a+b}{2}\right)\right|^{q} \frac{s-4 \varepsilon-2 s \varepsilon+2(2 \varepsilon)^{s+2}+1}{(s+1)(s+2)}+\left|f^{\prime}(b)\right|^{q} \frac{2(1-2 \varepsilon)^{s+2}+4 \varepsilon+2 s \varepsilon-1}{(s+1)(s+2)}\right)^{\frac{1}{q}}\right]
\end{aligned}
$$

where

$$
\begin{aligned}
\int_{0}^{1}|t-2 \varepsilon| d t & =\int_{0}^{2 \varepsilon}(2 \varepsilon-t) d t+\int_{2 \varepsilon}^{1}(t-2 \varepsilon) d t=4 \varepsilon^{2}-2 \varepsilon+\frac{1}{2} \\
\int_{0}^{2 \varepsilon} t^{s}(2 \varepsilon-t) d t & =\frac{(2 \varepsilon)^{s+2}}{(s+1)(s+2)} \\
\int_{0}^{2 \varepsilon}(2 \varepsilon-t)(1-t)^{s} d t & =\frac{(1-2 \varepsilon)^{s+2}+4 \varepsilon+2 s \varepsilon-1}{(s+1)(s+2)} \\
\int_{2 \varepsilon}^{1} t^{s}(t-2 \varepsilon) d t & =\frac{s-4 \varepsilon-2 s \varepsilon+(2 \varepsilon)^{s+2}+1}{(s+1)(s+2)} \\
\int_{2 \varepsilon}^{1}(t-2 \varepsilon)(1-t)^{s} d t & =\frac{(1-2 \varepsilon)^{s+2}}{(s+1)(s+2)} .
\end{aligned}
$$

In case $\frac{1}{2} \leq \varepsilon \leq 1$, by Lemma 1 and using the Hölder inequality, we have

$$
\begin{aligned}
& \left|(1-2 \varepsilon) f\left(\frac{a+b}{2}\right)+\varepsilon[f(a)+f(b)]-\frac{1}{b-a} \int_{a}^{b} f(x) d x\right| \\
& \leq \frac{b-a}{4}\left[\left(\int_{0}^{1}|t-2 \varepsilon| d t\right)^{1-\frac{1}{q}}\left(\int_{0}^{1}|t-2 \varepsilon|\left|f^{\prime}\left(t \frac{a+b}{2}+(1-t) a\right)\right|^{q} d t\right)^{\frac{1}{q}}\right. \\
& \left.\quad+\left(\int_{0}^{1}|2 \varepsilon-t| d t\right)^{1-\frac{1}{q}}\left(\int_{0}^{1}|2 \varepsilon-t|\left|f^{\prime}\left(t \frac{a+b}{2}+(1-t) b\right)\right|^{q} d t\right)^{\frac{1}{q}}\right]
\end{aligned}
$$




$$
\begin{aligned}
\leq & \frac{b-a}{4}\left(2 \varepsilon-\frac{1}{2}\right)^{1-\frac{1}{q}}\left[\left(\int_{0}^{1}|2 \varepsilon-t|\left(t^{s}\left|f^{\prime}\left(\frac{a+b}{2}\right)\right|^{q}+(1-t)^{s}\left|f^{\prime}(a)\right|^{q}\right) d t\right)^{\frac{1}{q}}\right. \\
& \left.+\left(\int_{0}^{1}|2 \varepsilon-t|\left(t^{s}\left|f^{\prime}\left(\frac{a+b}{2}\right)\right|^{q}+(1-t)^{s}\left|f^{\prime}(b)\right|^{q}\right) d t\right)^{\frac{1}{q}}\right] \\
= & \frac{b-a}{4}\left(2 \varepsilon-\frac{1}{2}\right)^{1-\frac{1}{q}}\left[\left(\left|f^{\prime}\left(\frac{a+b}{2}\right)\right|^{q} \int_{0}^{1}(2 \varepsilon-t) t^{s} d t+\left|f^{\prime}(a)\right|^{q} \int_{0}^{1}(2 \varepsilon-t)(1-t)^{s} d t\right)^{\frac{1}{q}}\right. \\
& \left.+\left(\left|f^{\prime}\left(\frac{a+b}{2}\right)\right|^{q} \int_{0}^{1}(2 \varepsilon-t) t^{s} d t+\left|f^{\prime}(b)\right|^{q} \int_{0}^{1}(2 \varepsilon-t)(1-t)^{s} d t\right)^{\frac{1}{q}}\right] \\
= & \frac{b-a}{4}\left(2 \varepsilon-\frac{1}{2}\right)^{1-\frac{1}{q}}\left[\left(\left|f^{\prime}\left(\frac{a+b}{2}\right)\right|^{q} \frac{2 \varepsilon(s+2)-(s+1)}{(s+1)(s+2)}+\left|f^{\prime}(a)\right|^{q} \frac{4 \varepsilon+2 s \varepsilon-1}{(s+1)(s+2)}\right)^{\frac{1}{q}}\right. \\
& \left.+\left(\left|f^{\prime}\left(\frac{a+b}{2}\right)\right|^{q} \frac{2 \varepsilon(s+2)-(s+1)}{(s+1)(s+2)}+\left|f^{\prime}(b)\right|^{q} \frac{4 \varepsilon+2 s \varepsilon-1}{(s+1)(s+2)}\right)^{\frac{1}{q}}\right] .
\end{aligned}
$$

The proof is completed.

Corollary 3. Let $I \subset[0, \infty), f: I \rightarrow R$ be a differentiable function on $I^{\circ}$ such that $f^{\prime} \in L^{1}[a, b]$, where $a, b \in I$ with $a<b$. If $\left|f^{\prime}\right|$ is s-convex in the second sense on $[a, b]$ for some fixed $s \in(0,1]$, then the following inequalities hold:

$$
\begin{aligned}
\mid(1-2 \varepsilon) f\left(\frac{a+b}{2}\right) & +\varepsilon[f(a)+f(b)]-\frac{1}{b-a} \int_{a}^{b} f(x) d x \mid \\
& \leq \frac{b-a}{4}\left(2\left|f^{\prime}\left(\frac{a+b}{2}\right)\right| P+\left(\left|f^{\prime}(a)\right|+\left|f^{\prime}(b)\right|\right) Q\right)
\end{aligned}
$$

for $0 \leq \varepsilon \leq \frac{1}{2}$, where $P=\frac{s-4 \varepsilon-2 s \varepsilon+2(2 \varepsilon)^{s+2}+1}{(s+1)(s+2)}, Q=\frac{2(1-2 \varepsilon)^{s+2}+4 \varepsilon+2 s \varepsilon-1}{(s+1)(s+2)}$, and

$$
\begin{aligned}
\mid(1-2 \varepsilon) f\left(\frac{a+b}{2}\right) & +\varepsilon[f(a)+f(b)]-\frac{1}{b-a} \int_{a}^{b} f(x) d x \mid \\
& \leq \frac{b-a}{4} 2\left(\left|f^{\prime}\left(\frac{a+b}{2}\right)\right| U+\left(\left|f^{\prime}(a)\right|+\left|f^{\prime}(b)\right|\right) V\right)
\end{aligned}
$$

for $\frac{1}{2} \leq \varepsilon \leq 1$ where $U=\frac{2 \varepsilon(s+2)-(s+1)}{(s+1)(s+2)}, V=\frac{4 \varepsilon+2 s \varepsilon-1}{(s+1)(s+2)}$.

Proof. Inequalities (2.11) and (2.12) are immediate by setting $q=1$ in (2.9) and (2.10) of Corollary 2. 
Remark 2. If we take $\varepsilon=0$ in (2.11), then we get a midpoint type inequality $\left|\frac{1}{b-a} \int_{a}^{b} f(x) d x-f\left(\frac{a+b}{2}\right)\right| \leq \frac{b-a}{4}\left(2\left|f^{\prime}\left(\frac{a+b}{2}\right)\right| \frac{1}{s+2}+\frac{\left|f^{\prime}(a)\right|+\left|f^{\prime}(b)\right|}{(s+1)(s+2)}\right)$.

If we take $\varepsilon=\frac{1}{2}$ in (2.11) or (2.12), then we get a trapezoid type inequality

$$
\begin{aligned}
& \left|\frac{1}{b-a} \int_{a}^{b} f(x) d x-\frac{f(a)+f(b)}{2}\right| \\
& \quad \leq \frac{b-a}{4}\left(2\left|f^{\prime}\left(\frac{a+b}{2}\right)\right| \frac{1}{(s+1)(s+2)}+\frac{\left(\left|f^{\prime}(a)\right|+\left|f^{\prime}(b)\right|\right)}{(s+2)}\right) .
\end{aligned}
$$

If we take $\varepsilon=\frac{1}{4}$ in (2.11), then we get a Bullen type inequality

$$
\begin{aligned}
& \left|\frac{1}{b-a} \int_{a}^{b} f(x) d x-\frac{1}{4}\left[f(a)+2 f\left(\frac{a+b}{2}\right)+f(b)\right]\right| \\
& \leq \frac{b-a}{4}\left(\left|f^{\prime}\left(\frac{a+b}{2}\right)\right| \frac{s+2^{-s}}{(s+1)(s+2)}+\left(\left|f^{\prime}(a)\right|+\left|f^{\prime}(b)\right|\right) \frac{s+2^{-s}}{2(s+1)(s+2)}\right) .
\end{aligned}
$$

If we take $\varepsilon=\frac{1}{6}$ in (2.11), then we get a Simpson type inequality

$$
\begin{aligned}
& \left|\frac{1}{b-a} \int_{a}^{b} f(x) d x-\frac{1}{6}\left[f(a)+4\left(\frac{a+b}{2}\right)+f(b)\right]\right| \\
& \leq \frac{b-a}{4}\left(2\left|f^{\prime}\left(\frac{a+b}{2}\right)\right| \frac{2 s+2 \times 3^{s+1}+1}{3(s+1)(s+2)}+\left(\left|f^{\prime}(a)\right|+\left|f^{\prime}(b)\right|\right) \frac{s+2^{s+3} \times 3^{-s-1}-1}{3(s+1)(s+2)}\right) .
\end{aligned}
$$

Remark 3. If we put $M=\sup _{x \in[a, b]}\left|f^{\prime}\right|$ in (2.13)-(2.16), then we have

$$
\begin{aligned}
\left|\frac{1}{b-a} \int_{a}^{b} f(x) d x-f\left(\frac{a+b}{2}\right)\right| & \leq \frac{b-a}{2} \frac{M}{s+1} \\
\left|\frac{1}{b-a} \int_{a}^{b} f(x) d x-\frac{f(a)+f(b)}{2}\right| & \leq \frac{b-a}{2} \frac{M}{s+1} \\
\left|\frac{1}{b-a} \int_{a}^{b} f(x) d x-\frac{1}{4}\left[f(a)+2 f\left(\frac{a+b}{2}\right)+f(b)\right]\right| & \leq \frac{b-a}{2}\left(M \frac{s+2^{-s}}{(s+1)(s+2)}\right)
\end{aligned}
$$




$$
\begin{aligned}
\mid \frac{1}{b-a} \int_{a}^{b} f(x) d x-\frac{1}{6}[f(a) & \left.+4\left(\frac{a+b}{2}\right)+f(b)\right] \mid \\
\leq & M \frac{b-a}{6}\left(\frac{3 s+2 \times 3^{s+1}}{(s+1)(s+2)}+\frac{2^{s+3} \times 3^{-s-1}}{(s+1)(s+2)}\right) .
\end{aligned}
$$

Remark 4. If we further take $s=1$ in (2.17)-(2.20) for functions $f$ with convex $\left|f^{\prime}\right|$, we have

$$
\begin{aligned}
\left|\frac{1}{b-a} \int_{a}^{b} f(x) d x-f\left(\frac{a+b}{2}\right)\right| & \leq \frac{M(b-a)}{4} \\
\left|\frac{1}{b-a} \int_{a}^{b} f(x) d x-\frac{f(a)+f(b)}{2}\right| & \leq \frac{M(b-a)}{4} \\
\left|\frac{1}{b-a} \int_{a}^{b} f(x) d x-\frac{1}{4}\left[f(a)+2 f\left(\frac{a+b}{2}\right)+f(b)\right]\right| & \leq \frac{M(b-a)}{8} \\
\left|\frac{1}{b-a} \int_{a}^{b} f(x) d x-\frac{1}{6}\left[f(a)+4\left(\frac{a+b}{2}\right)+f(b)\right]\right| & \leq \frac{205 M(b-a)}{324} .
\end{aligned}
$$

Corollary 4. Under the assumption of Theorem 1 , if $\left|f^{\prime}\right|^{q}$ is $P(I)$, then the following inequality holds:

$$
\begin{aligned}
& \left|(1-2 \varepsilon) f\left(\frac{a+b}{2}\right)+\varepsilon[f(a)+f(b)]-\frac{1}{b-a} \int_{a}^{b} f(x) d x\right| \\
& \leq \frac{b-a}{4}\left(4 \varepsilon^{2}-2 \varepsilon+\frac{1}{2}\right)\left[\left(\left|f^{\prime}\left(\frac{a+b}{2}\right)\right|^{q}+\left|f^{\prime}(a)\right|^{q}\right)^{\frac{1}{q}}+\left(\left|f^{\prime}\left(\frac{a+b}{2}\right)\right|^{q}+\left|f^{\prime}(b)\right|^{q}\right)^{\frac{1}{q}}\right]
\end{aligned}
$$

for $0 \leq \varepsilon \leq \frac{1}{2}$,

$$
\begin{aligned}
& \left|(1-2 \varepsilon) f\left(\frac{a+b}{2}\right)+\varepsilon[f(a)+f(b)]-\frac{1}{b-a} \int_{a}^{b} f(x) d x\right| \\
& \leq \frac{b-a}{4}\left(2 \varepsilon-\frac{1}{2}\right)\left[\left(\left|f^{\prime}\left(\frac{a+b}{2}\right)\right|^{q}+\left|f^{\prime}(a)\right|^{q}\right)^{\frac{1}{q}}+\left(\left|f^{\prime}\left(\frac{a+b}{2}\right)\right|^{q}+\left|f^{\prime}(b)\right|^{q}\right)^{\frac{1}{q}}\right]
\end{aligned}
$$

for $\frac{1}{2} \leq \varepsilon \leq 1$.

Proof. Proof of inequalities (2.25) and (2.26) is explicit by choosing $h(t)=1$ in (2.6) and (2.7) of Theorem 1. 
Corollary 5. Under the assumption of Theorem 1 , if $\left|f^{\prime}\right|^{q}$ is tgs-convex, then the following inequality holds:

$$
\begin{aligned}
& \left|(1-2 \varepsilon) f\left(\frac{a+b}{2}\right)+\varepsilon[f(a)+f(b)]-\frac{1}{b-a} \int_{a}^{b} f(x) d x\right| \\
& \leq \frac{b-a}{4}\left(4 \varepsilon^{2}-2 \varepsilon+\frac{1}{2}\right)^{1-\frac{1}{q}}\left(\frac{(1-4 \varepsilon)}{12}+\frac{8 \varepsilon^{3}(1-\varepsilon)}{3}\right)^{\frac{1}{q}} \\
& \quad \times\left[\left(\left|f^{\prime}\left(\frac{a+b}{2}\right)\right|^{q}+\left|f^{\prime}(a)\right|^{q}\right)^{\frac{1}{q}}+\left(\left|f^{\prime}\left(\frac{a+b}{2}\right)\right|^{q}+\left|f^{\prime}(b)\right|^{q}\right)^{\frac{1}{q}}\right],
\end{aligned}
$$

for $0 \leq \varepsilon \leq \frac{1}{2}$, and

$$
\begin{aligned}
& \left|(1-2 \varepsilon) f\left(\frac{a+b}{2}\right)+\varepsilon[f(a)+f(b)]-\frac{1}{b-a} \int_{a}^{b} f(x) d x\right| \\
& \leq \frac{b-a}{8 \times 6^{1 / q}}(4 \varepsilon-1)\left[\left(\left|f^{\prime}\left(\frac{a+b}{2}\right)\right|^{q}+\left|f^{\prime}(a)\right|^{q}\right)^{\frac{1}{q}}+\left(\left|f^{\prime}(b)\right|^{q}+\left|f^{\prime}\left(\frac{a+b}{2}\right)\right|^{q}\right)^{\frac{1}{q}}\right],
\end{aligned}
$$

for $\frac{1}{2} \leq \varepsilon \leq 1$.

Proof. Proof of inequalities (2.27) and (2.28) is explicit by taking $h(t)=t(1-t)$ in (2.6) and (2.7) of Theorem 1.

\section{ApPliCATIONS}

We consider the means for arbitrary positive numbers $a, b(a \neq b)$ as follows: The arithmetic mean:

the generalized log-mean:

$$
A(a, b)=\frac{a+b}{2}
$$

$$
L_{p}(a, b)=\left[\frac{b^{p+1}-a^{p+1}}{(p+1)(b-a)}\right]^{\frac{1}{p}}, p \in \mathbb{R} \backslash\{-1,0\} .
$$

Now, by using the result of the second section, we give some applications to special means of real numbers.

Proposition 1. Let $0<a<b, s \in(0,1)$. Then the following inequalities hold:

$$
\begin{aligned}
\left|L_{s}^{s}(a, b)-A^{s}(a, b)\right| & \leq \frac{s(b-a)}{2}\left(\frac{A^{s}(a, b)}{s+2}+\frac{A\left(a^{s}, b^{s}\right)}{(s+1)(s+2)}\right) \\
\left|L_{s}^{s}(a, b)-A\left(a^{s}, b^{s}\right)\right| & \leq \frac{s(b-a)}{2}\left(\frac{A^{s}(a, b)}{(s+1)(s+2)}+\frac{A\left(a^{s}, b^{s}\right)}{s+2}\right)
\end{aligned}
$$




$$
\begin{aligned}
& \left|L_{s}^{s}(a, b)-\frac{A^{s}(a, b)+A\left(a^{s}, b^{s}\right)}{2}\right| \leq \frac{s(b-a)}{4} \frac{s+2^{-s}}{(s+1)(s+2)}\left(A^{s}(a, b)+2 A\left(a^{s}, b^{s}\right)\right) \\
& \left|L_{s}^{s}(a, b)-\frac{2 A^{s}(a, b)+A\left(a^{s}, b^{s}\right)}{3}\right| \\
& \leq \frac{s(b-a)}{6}\left(A^{s}(a, b) \frac{2 s+2 \times 3^{s+1}+1}{(s+1)(s+2)}+A\left(a^{s}, b^{s}\right) \frac{s+2^{s+3} \times 3^{-s-1}-1}{(s+1)(s+2)}\right)
\end{aligned}
$$

Proof. The inequalities are derived from (2.13)-(2.16) applied to the $s$-convex functions $f: \mathbb{R} \rightarrow \mathbb{R}, f(x)=x^{s}, s \in(0,1), x \in[a, b]$ and $f^{\prime}(x)=s x^{s-1}, s \in(0,1)$, $x \in[a, b]$. The details are disregarded.

Proposition 2. Let $0<a<b, s \in(0,1)$. Then the following inequalities hold:

$$
\begin{aligned}
\left|L_{s}^{s}(a, b)-A^{s}(a, b)\right| & \leq \frac{(b-a)(s+2)}{2 a^{1-s}} \\
\left|L_{s}^{s}(a, b)-A\left(a^{s}, b^{s}\right)\right| & \leq \frac{(b-a)(s+2)}{2 a^{1-s}} \\
\left|L_{s}^{s}(a, b)-\frac{A^{s}(a, b)+A\left(a^{s}, b^{s}\right)}{2}\right| & \leq \frac{b-a}{2} \frac{s+2^{-s}}{a^{1-s}} \\
\left|L_{s}^{s}(a, b)-\frac{2 A^{s}(a, b)+A\left(a^{s}, b^{s}\right)}{3}\right| & \leq \frac{b-a}{6 a^{1-s}}\left(3 s+2 \times 3^{s+1}+2^{s+3} \times 3^{-s-1}\right) .
\end{aligned}
$$

Proof. The inequalities are derived from (2.17)-(2.20) applied to the $s$-convex functions $f: \mathbb{R} \rightarrow \mathbb{R}, f(x)=x^{s}, s \in(0,1), x \in[a, b]$ and $f^{\prime}(x)=s x^{s-1}, s \in(0,1)$, $x \in[a, b]$ and we might take $M=\frac{(s+1)(s+2)}{a^{1-s}}$. The details are disregarded.

\section{REFERENCES}

[1] H. Alzer, "A superadditive property of Hadamard's gamma function," Abh. Math. Semin. Univ. Hambg., vol. 79, pp. 11-23, 2009, doi: 10.1007/s12188-008-0009-5.

[2] M. Bessenyei, "The Hermite-Hadamard inequality in Beckenbach's setting." J. Math. Anal. Appl.., vol. 364, no. 2, pp. 366-383, 2010, doi: 10.1016/j.jmaa.2009.11.015.

[3] M. Bessenyei and Z. Páles, "Higher-order generalizations of Hadamard's inequality." Publ. Math. Debrecen., vol. 61, no. 34, pp. 623-643, 2002.

[4] M. Bessenyei and Z. Páles, "Characterizations of convexity via Hadamard's inequality." Math. Inequal. Appl., vol. 9, no. 1, pp. 53-62, 2006, doi: 10.7153/mia-09-06.

[5] M. Bombardelli and S. Varošanec, " Properties of $h$-convex functions related to the HermiteHadamard-Fejér inequalities.” Comput. Math. Appl., vol. 58, pp. 1869-1877, 2009, doi: 10.1016/j.camwa.2009.07.073.

[6] W. W. Breckner, "Stetigkeitsaussagen für eine Klasse verallgemeinerter konvexer Funktionen in topologischen linearen Raumen.” Pupl. Inst. Math., vol. 23, pp. 13-20, 1978.

[7] W. W. Breckner, "Continuity of generalized convex and generalized concave set-valued functions.” Rev Anal. Numer. Thkor. Approx., vol. 22, pp. 39-51, 1993. 
[8] P. Burai and A. Házy, "On approximately $h$-convex functions.” J. Convex Anal., vol. 18, no. 2, pp. 447-454, 2011.

[9] P. Burai, A. Házy, and T. Juhász, "Bernstein-Doetsch type results for $s-$ convex functions." Publ. Math. Debrecen., vol. 75, no. 1-2, pp. 23-31, 2009.

[10] P. Burai, A. Házy, and T. Juhász, "On approximately Breckner $s$-convex functions." Control Cybernet., vol. 40, no. 1, pp. 91-99, 2011.

[11] S. S. Dragomir, R. P. Agarwal, and P. Cerone, "On Simpson's inequality and applications." J. Inequal. Appl., vol. 5, no. 6, pp. 533-579, 2000, doi: 10.1155/S102558340000031X.

[12] S. S. Dragomir, J. Pečarić, and L. E. Persson, "Some inequalities of Hadamard type." Soochow J.Math., vol. 21, pp. 235-241, 1995.

[13] E. K. Godunova and V. I. Levin, "Neravenstva dlja funkcii sirokogo klassa, soderzascego vypuklye, monotonnye i nekotorye drugie vidy funkii." Vycislitel. Mat. i. Fiz. Mezvuzov. Sb. Nauc. Trudov, MGPI. Moskva, pp. 138-142, 1985.

[14] H. Hudzik and L. Maligranda, "Some remarks on $s$-convex functions." Aequationes Math., vol. 48, pp. 100-111, 1994, doi: 10.1007/BF01837981.

[15] J. Makó and Z. Páles, “Implications between approximate convexity properties and approximate Hermite-Hadamard inequalities," Cent. Eur. J. Math., vol. 10, no. 3, pp. 1017-1041, 2012, doi: 10.2478/s11533-012-0027-5.

[16] J. Makó and Z. Páles, "Approximate Hermite-Hadamard type inequalities for approximately convex functions," Math. Inequal. Appl., vol. 16, no. 2, pp. 507-526, 2013, doi: 10.7153/mia-16-37.

[17] M. E. Özdemir, M. Gürbüz, and A. O. Akdemir, "Inequalities for $h$-convex functions via further properties.” RGMIA Research Report Collection., vol. 14, no. 22, 2011.

[18] J. Pečarić, F. Proschan, and Y. Tong, Convex Functions, Partial Orderings and Statistical Applications. Academic Press, 1991.

[19] M. Z. Sarikaya, A. Saglam, and H.Yildirim, "On some Hadamard-type inequalities for $h$-convex functions." J. Math. Inequal., vol. 2, no. 3, pp. 335-341, 2008, doi: 10.7153/jmi-02-30.

[20] M. Tunc, E. Gov, and U. Sanal, "On tgs-convex functions and their inequalities," Facta Universitatis Ser. Math. Inform., vol. 30, no. 5, pp. 679-691, 2015.

[21] M. Tunc and H. Yildirim, "On MT-convexity," http://arxiv.org/pdf/1205.5453.pdf, preprint.

[22] S. Varošanec, "On $h$-convexity." J. Math. Anal. Appl., vol. 326, no. 1, pp. 303-311, 2007, doi: 10.1016/j.jmaa.2006.02.086.

Authors' addresses

Musa Çakmak

Mustafa Kemal University, Yaladağ 1 Vocational School, Hatay, Turkey

E-mail address: enkucukcakmak@gmail.com

\section{Mevlüt Tunç}

Mustafa Kemal University, Faculty of Science and Arts, Department of Mathematics, Hatay, Turkey

E-mail address: mevluttttunc@gmail.com

\section{Ayşegül Acem}

Mustafa Kemal University, Institute of Science, Department of Informatics, Hatay, Turkey

E-mail address: ayseguldurgun0708@gmail.com 